\title{
Pandemic Control COVID 19: The Results of Analytical Studies of Ten Health Systems in the Word. Scopus Review
}

\section{Zaadoud B rahim}

Department of Biophysics and Clinical MRI Methods, Clinical Neurosciences Laboratory, University of Fez, Ministry of health, Morocco

Correspondence should be addressed to Zaadoud Brahim, zaadoud@gmail.com

Received: June 08, 2020; Accepted: June 21, 2020; Published: June 28, 2020

\begin{abstract}
$\underline{\text { ABSTRACT }}$
OBJECTIVES

Description of the epidemiological situation of COVID-19 and analyze and compare between ten-health systems control epidemic around the word for use in public health system settings.
\end{abstract}

\section{METHODS}

This study was a descriptive, exploratory analysis of all cases of COVID-19 in the world especially in the ten countries. We conducted a Scopus review of the literature. Thirty articles are been recolted.

\section{RESULTS}

The estimates of human transmissibility (R0) range from 2 to 3. Pre-symptomatic transmission has been reported. The Incubation period is 5.1 days; the overall global case fatality rate is currently approximately $6 \%$. The countries are characterized into three categories of pandemic control: excellent level of control for Germany, South Korea, Hong Kong and turkey a medium level for China, Morocco and a low level for the USA, France, Spain and Italy.

\section{CONCLUSION}

The present descriptive, exploratory analysis offers important new information to the international community on the Corona virus Pandemic in world. Important questions remain including determination of infectiousness period, identification of transmission routes, and effective treatment and prevention methods including further test development, drug development, and vaccine development.

\section{KEYWORDS}

COVID 19; Pandemic; Health systems; Control diseases

Citation: Zaadoud Brahim, Pandemic Control COVID 19: The Results of Analytical Studies of Ten Health Sy stems in the Word. Scopus Review. J Clin Cases Rep 3(S4): 3-8. 


\section{INTRODUCTION}

In the past two decades two highly pathogenic human coronaviruses, responsible for severe acute respiratory syndrome (SARS-Cov) and responsible for Middle East respiratory syndrome (MERS-Cov), [1,2] have emerged in two separate events. A recent emergence, rapid kinetics around the world and a "Severe" Infection noticed On 29 December 2019 in China a clustering of cases of unusual pneumonia with an apparent link to a market that sells live fish, poultry and animals to the public [3]. Officially named coronavirus 2 (SARS-Cov2). On 30 January 2020, the World Health Organization declared that the outbreak of SARS-Cov-2 constituted a public health emergency of International concern.

\section{EPIDEMIOLOGY}

The Chinese Centers for Disease Control (CDC) recently reported that most of the confirmed cases were classified as mild or moderate, $13.8 \%$ as severe, and only $4.7 \%$ as critically ill. [4]. Advanced age (>60), male sex, and comorbidities (particularly hypertension) are believed to be risk factors for severe disease and death from SARSCov-2 infection. Male sex was more predominant in patients who died than in those who recovered. Chronic hypertension and other cardiovascular comorbidities were more frequent among deceased patients than recovered patients [5]. Wang and al, report a relatively high mortality rate for COVID-19 of up to $14.1 \%$, which is higher than in recent reports [6]. In accordance with the recent reports on characteristics of patients with COVID-19 who needed management in intensive care units [6-8]. The median estimated incubation period is five to six days (range 0 days to 14 days) [9]. The median age of patients with a confirmed case is around 59 years [10]. In adults, the most common symptoms at presentation are cough (68\%), fever (44\%), fatigue (38\%), myalgia/arthralgia (15\%), and headache (14\%) [10]. Initial data indicate that more than $80 \%$ of patients have asymptomatic to moderate disease and recover, but about $15 \%$ may get severe disease including pneumonia, and around 5\% become critically unwell with septic shock and/or multi-organ and respiratory failure [11].

\section{METHOD}

Study design

This study was a descriptive, exploratory analysis of all cases of COVID-19. We have opted for a comparative and analytical approach to 10 data countries.

\section{Pandemic control criteria}

We have integrated the elements of primary and secondary prevention as input to the health systems and efficiency criteria as output.

\section{Population data}

Descriptive epidemiological indicators case number, death and recovered and prevalence, Number of new cases (Incidence); Case fatality ratio (CFR), Attack rate; Basic reproduction rate (R0); Generation interval.

The management of the epidemic was very different between the three groups in Asia (group A); the European countries and the USA (group B) and Morocco (Group C). the criteria for choosing countries was a reasoned choice to encompass the different control strategies and the continents most affected by COVID 19 and a representativeness of the continents especially for Morocco which represents the African continent and the developing countries. In Group 'A' for East Asian countries (Chine, South Korea and Hong Kong), which have mobilized effectively against the pandemic: Targeted containment not generalized Good hygiene, screening at will, monitoring and geolocalization of contaminated persons-Wearing a protective face mask is mandatory in public places. In Group 'B' Europe countries and United States (Italy, Spain, France, Germany, United Kingdom and Turkey), most of the containment decisions announced by the governments of 
these countries were taken late and Group ' $C$ ' for Morocco.

\section{The analysis}

Confirmed cases, demographic and clinical characteristics were summarized using descriptive statistics. Case fatality rates were calculated as the total number of deaths (numerator) divided by the total number of cases (denominator), expressed as a percent. Will be presented in the form of static and dynamic comparative tables analysing the results in focus of the interventions carried out with effectiveness indicators (Deaths/1 million population, Critical care beds 100000 population, Hospital overcrowding, Research investment). The ranking of countries is based on the sum of scores from one to three assigned to each variable.

\section{Data source}

31 Articles and report written on COVID 19 until April $15,2020$.

\section{RESULTS}

\section{Transmission and dynamic of transmissibility}

Recent epidemiological reports have provided evidence for person-to-person transmission of the SARS-Cov-2 in family and hospital settings [10,12].

\section{Transmissibility (RO) range}

An infectious disease outbreak can be characterized by its reproductive number 'R0'. Early estimates of the attack rate in China range from $3 \%$ - 10\%, mainly in households [13]. The current estimates of R0 for the SARS-CoV-2 outbreak in China range from 2.5 to 2.9, with an associated all-age case fatality ratio estimated to be $2.3 \%$ [14]. DHS Science and Technology High-quality estimates of human transmissibility (R0) range from 2.2 to 3 [15]. SARS-CoV-2 is believed to spread through close contact and droplet transmission, with fomite transmission [6]. Pre-symptomatic or asymptomatic patients can transmit SARS-CoV-2; between $12 \%$ and $23 \%$ of infections may be caused by asymptomatic or pre-symptomatic transmission [16]. Modellers have suggested reproductive rates (R0) of 3.8 (95\% confidence interval, 3.6 - 4.0) [17] and 2.6 (1.5 - 3.5) [18].

\section{Clinical and symptoms}

\section{Asymptomatic transmission}

Pre-symptomatic transmission has been reported; exposure in these cases occurred 1 days - 3 days before the source patient developed symptoms [19].

There is some evidence that spread from asymptomatic carriers is possible, although it is thought that transmission is greatest when people are symptomatic [20]. Estimating the prevalence of asymptomatic cases in the population is difficult. A modelling study found that approximately 700 people with confirmed infection (18\%) were asymptomatic [21]. A recent modelling study suggested that asymptomatic individuals might be major drivers for the growth of the COVID-19 pandemic [19].

\section{Incubation period}

The best current estimate of the COVID-19 incubation period is 5.1 days, with $99 \%$ of individuals exhibiting symptoms within 14 days of exposure. Fewer than $2.5 \%$ of infected individuals show symptoms sooner than 2 days after exposure [22]. The reported range of incubation periods is wide, with high-end estimates of 11.3 and 18 days [10].

\section{Clinical symptoms}

Individuals can test positive for COVID-19 despite lacking clinical symptoms. Individuals can be infectious while asymptomatic [23] and asymptomatic individuals can have similar amounts of virus in their nose and throat as symptomatic individuals. Infectious period is unknown, but possibly up to 10 days - 14 days [24]. Experimentally infected macaques were not capable of being reinfected after their primary infection resolved [25]. 


\section{Clinical diagnosis}

PCR protocols and primers have been widely shared among international researchers though PCR-based diagnostic assays do not differentiate between active and inactive virus [10].

\section{Mortality rate and contagiousness}

The overall global case fatality rate is currently approximately $6 \%$ based on World Health Organization data as of 13 April 2020. The case fatality rate varies between countries; for example, it is currently higher in countries such as France, Italy, and Spain, and lower in countries such as the US, Germany, and Australia. The case fatality rate is estimated at approximately $2 \%$ overall, but ranges from $0.2 \%$ in people under 50 to $14.8 \%$ in those over 80 , and is higher among those with chronic comorbid conditions [11] . Based on data from EU/EEA countries, $32 \%$ of the diagnosed cases have required hospitalization and $2.4 \%$ have had severe illness requiring respiratory support and/or ventilation. The crude fatality rate was $1.5 \%$ among diagnosed cases and $11 \%$ among hospitalized cases. The likelihood of hospitalization, severe illness and death increases in persons over 65 years of age and those with defined risk factors including hypertension, diabetes, cardiovascular disease, chronic respiratory disease, compromised immune status, cancer and obesity [26]. The confirmed case fatality ratio (CFR) is the total number of deaths divided by the total number of confirmed cases at one point in time appears quite constant oscillating between $2 \%-3 \%$ [27]. The case fatality rate was highest among critical cases $(49 \%)$, it was also higher in patients aged 80 years and older (15\%), males (2.8\% versus $1.7 \%$ for females), and patients with comorbidities (10.5\% for cardiovascular disease, $7.3 \%$ for diabetes, $6.3 \%$ for chronic respiratory disease, $6 \%$ for hypertension, and $5.6 \%$ for cancer) [28]. Another study found the case fatality rate in China to be $6.4 \%$ in patients aged $\geq 60$ years versus $0.32 \%$ in patients aged. In Italy, the case fatality rate was $8.5 \%$ in patients aged 60 years to 69 years, $35.5 \%$ in patients aged 70 yeras to 79 years, and $52.5 \%$ in patients aged $\geq 80$ years [29]. In a case series of 1591 critically ill patients in Lombardy, the majority of patients were older men, a large proportion required mechanical ventilation and high levels of positive endexpiratory pressure, and the mortality rate in the intensive care unit was $26 \%$ [30]. In the US, the case fatality rate was highest among patients aged $\geq 85$ years $(10 \%$ to $27 \%)$, followed by those aged 65 years to 84 years $(3 \%$ to $11 \%), 55$ years to 64 years ( $1 \%$ to $3 \%), 20$ years to 54 years $(<1 \%)$, and $\leq 19$ years (no deaths). Patients aged $\geq 65$ years accounted for $80 \%$ of deaths [26]. The results of the ten countries are summarized in a table 1. This (table 1) shows that there are several three categories of countries those with a very high contamination rate (+ 4000 cases/million) USA and Spain, those with a high rate (between 2000 and 4000) UK, Italy, France and Germany, those considered medium (between 1000 and 2000) Turkey and those with a low rate (-1000) China, South Korea, and Morocco.

\begin{tabular}{|c|c|c|c|c|c|c|}
\hline & $\begin{array}{c}\text { Total } \\
\text { Cases }\end{array}$ & $\begin{array}{c}\text { Tot } \\
\text { Cases/1m } \\
\text { Population }\end{array}$ & $\begin{array}{c}\text { Total } \\
\text { Deaths }\end{array}$ & $\begin{array}{c}\text { \% } \\
\text { Death/Cases }\end{array}$ & $\begin{array}{c}\text { Deaths/1 M } \\
\text { Population }\end{array}$ & $\begin{array}{c}\text { Test/1 M } \\
\text { Population }\end{array}$ \\
\hline USA & 1408039 & 4254 & 83366 & 5.92 & 252 & 30010 \\
\hline Spain & 269520 & 5765 & 26920 & 9.99 & 576 & 52781 \\
\hline UK & 226463 & 3336 & 32692 & 14.44 & 482 & 29566 \\
\hline Italy & 221216 & 3659 & 30911 & 13.97 & 511 & 44221 \\
\hline France & 178225 & 2730 & 26991 & 15.14 & 414 & 21213 \\
\hline Germany & 173171 & 2067 & 7738 & 4.47 & 92 & 32891 \\
\hline Turkey & 141475 & 1677 & 3894 & 2.75 & 46 & 17082 \\
\hline China & 82919 & 58 & 4633 & 5.59 & 3 & \\
\hline $\begin{array}{c}\text { South } \\
\text { Korea }\end{array}$ & 10936 & 213 & 258 & 2.36 & 5 & 13281 \\
\hline Morocco & 6418 & 174 & 188 & 2.93 & 5 & 1946 \\
\hline
\end{tabular}

Table 1: Evolution over time of cases, deaths and test in different countries.

The classification according to percentage of deaths/registered cases (-3\%) for South Korea, Turkey and Morocco; (3\% - 6\%) for China, USA and Germany; $(6 \%-9 \%)$ and $(+9 \%)$ for Spain, Italy, France and UK. The classification according to the number of tests per million inhabitants $(+40000)$ for Italy and Spain; between 30000 and 40000 for Germany; USA and UK; between 20000 and 30000 for France and (-20000) for Turkey, China; Morocco and South korea. It is noted that there is no correlation between the number of cases and deaths so there is a correlation between the number of tests 
performed and the number of deaths. Where other elements are needed to explain these results and especially the elements of the fight against the epidemic.

\section{CONCLUSION}

COVID-19 epidemic has spread very quickly. The present descriptive, exploratory analysis offers important new information to the international community on the Corona virus pandemic in world. Important questions remain including determination of infectiousness period, identification of transmission routes, and effective treatment and prevention methods including further test development, drug development, and vaccine development.

\section{REFERENCES}

1. Drosten C, Günther S, Preiser W, et al. (2003) Identification of a novel coronavirus in patients with severe acute respiratory syndrome. New England Journal of Medicine 348(20): 1967-1976.

2. Zaki AM, Van Boheemen S, Bestebroer TM, et al. (2012) Isolation of a novel coronavirus from a man with pneumonia in Saudi Arabia. New England Journal of Medicine 367(19): 1814-1820.

3. McCloskey B, Heymann DL (2020) SARS to novel coronavirus-old lessons and new lessons. Epidemiology \& Infection 148.

4. Januzzi JL, van Kimmenade R, Lainchbury J, et al. (2006) NT-proBNP testing for diagnosis and short-term prognosis in acute destabilized heart failure: An international pooled analysis of 1256 patients: The International Collaborative of NT-proBNP Study. European Heart Journal 27(3): 330-337.

5. Chen T, Wu D, Chen H, et al. (2020) Clinical characteristics of 113 deceased patients with coronavirus disease 2019: Retrospective study. BMJ 368.

6. Wang D, Hu B, Hu C, et al. (2020) Clinical characteristics of 138 hospitalized patients with 2019 novel coronavirus infected pneumonia in Wuhan, China. JAMA 323(11): 1061-1069.

7. Huang C, Wang Y, Li X, et al. (2020) Clinical features of patients infected with 2019 novel coronavirus in Wuhan, China. The Lancet 395(10223): 497-506.

8. Chen N, Zhou M, Dong X, et al. (2020) Epidemiological and clinical characteristics of 99 cases of 2019 novel coronavirus pneumonia in Wuhan, China: A descriptive study. The Lancet 395(10223): 507-513.

9. World Health Organization (2020) Coronavirus disease 2019 (COVID-19): Situation report: 29.

10. Li Q, Guan X, Wu P, et al. (2020) Early transmission dynamics in Wuhan, China, of novel coronavirus-infected pneumonia. New England Journal of Medicine.

11. Wu Z, McGoogan JM (2020) Characteristics of and important lessons from the coronavirus disease 2019 (COVID-19) outbreak in China: Summary of a report of 72,314 cases from the Chinese Center for Disease Control and Prevention. JAMA 323(13): 1239-1242.

12. Chan JFW, Yuan S, Kok KH, et al. (2020) A familial cluster of pneumonia associated with the 2019 novel coronavirus indicating person-to-pers on transmission: A study of a family cluster. The Lancet 395(10223): 514-523.

13. Liu P, Beeler P, Chakrabarty RK (2020) COVID-19 progression timeline and effectiveness of response-to-Spread interventions across the United States. medRxiv.

14. Wu JT, Leung K, Leung GM (2020) Nowcasting and forecasting the potential domestic and International spread of the 2019-nCoV outbreak originating in Wuhan, China: A modelling study. The Lancet 395(10225): 689-697.

15. Xie J, Tong Z, Guan X, et al. (2020) Critical care crisis and some recommendations during the COVID-19 epidemic in China. Intensive Care Medicine: 1-4. 
http://www.tridhascholars.org | December-2020

16. Emami A, Javanmardi F, Pirbonyeh N, et al. (2020) Prevalence of underlying diseases in hospitalized patients with COVID-19: A systematic review and meta-analysis. Archives of Academic Emergency Medicine 8(1): e35.

17. Read JM, Bridgen JR, Cummings DA, et al. (2020) Novel coronavirus 2019-nCoV: Early estimation of epidemiological parameters and epidemic predictions. MedRxiv.

18. Imai N, Cori A, Dorigatti I, et al. (2020) Report 3: Transmissibility of 2019-nCoV. Imperial College London.

19. Aguilar JB, Faust JS, Westafer LM, et al. (2020) Investigating the impact of asymptomatic carriers on COVID-19 transmis sion. medRxiv.

20. Li C, Ji F, Wang L, et al. (2020) Asymptomatic and human-to-human transmission of SARS-CoV-2 in a 2-family cluster, Xuzhou, China. Emerging Infectious Diseases 26(7).

21. Mizumoto K, Kagaya K, Zarebski A, et al. (2020) Estimating the asymptomatic proportion of coronavirus disease 2019 (COVID-19) cases on board the Diamond Princess cruise ship, Yokohama, Japan, 2020. Eurosurveillance 25(10): 2000180.

22. Lauer SA, Grantz KH, Bi Q, et al. (2020) The incubation period of coronavirus disease 2019 (COVID-19) from publicly reported confirmed cases: Estimation and application. Annals of Internal Medicine 172(9): 577-582.

23. Tong ZD, Tang A, Li KF, et al. (2020) Potential presymptomatic transmission of SARS-CoV-2, Zhejiang province, China, 2020. Emerging Infectious Diseases 26(5): 1052.

24. Li R, Pei S, Chen B, et al. (2020) Substantial undocumented infection facilitates the rapid dissemination of novel coronavirus (SARS-CoV-2). Science 368(6490): 489-493.

25. Bao L, Deng W, Gao H, et al. (2020) Reinfection could not occur in SARS-CoV-2 infected rhesus macaques. BioRxiv.

26. Assessment RR (2020) Coronavirus disease 2019 (COVID-19) in the EU/EEA and the UK-ninth update.

27. Dente S (2020) Corona virus (COVID-19) epidemic A stock-flow perspective.

28. Sorbello M, El-Boghdadly K, Di Giacinto I, et al. (2020) The Italian COVID-19 Outbreak: Experiences and recommendations from clinical practice. Anaesthesia 75(6): 724-732.

29. Covid CDC, Team R (2020) Severe outcomes among patients with coronavirus disease 2019 (COVID-19)-United States, February 12-March 16, 2020. Morbidity and Mortality Weekly Report 69(12): 343-346.

30. Grasselli G, Zangrillo A, Zanella A, et al. (2020) Baseline characteristics and outcomes of 1591 patients infected with SARS-CoV-2 admitted to ICUs of the Lombardy Region, Italy. JAMA 323(16): 1574-1581. 